\title{
22 \\ Call Processing Architecture and Algorithms for Future Network
}

\author{
Geonung Kim, Sunshin An \\ Department of Electronic Engineering \\ Korea University \\ 1, 5-ka, Anam-dong, Sungbuk-ku, SEOUL, 136-701, KOREA \\ Phone: +82-2-920-1556, Fax: +82-2-928-0179 \\ E-mail:\{kgu,sunshin\}@dsys.korea.ac.kr
}

\begin{abstract}
This paper presents Service Call Processing Architecture 1 (SCPA1); a generic service call processing architecture that is well adaptable to emerging B-ISDN environment, especially to the Layer Network. We divide the call processing into two parts; one for negotiation among users and the other for management of lower layer resources. The Call Session Manager (CSM) is responsible for the negotiation among participants and the Resource Manager (RM) performs resource management functions. The recursive layering of CSM and RM makes it possible to model the Layer Network. We show SCPA1 is well suitable for the multiparty communication and the Layer Network. We describe the call processing algorithms of SCPA1 and depict the CSM functional model and RM functional model.
\end{abstract}

\section{Keywords}

Service Call Processing Architecture (SCPA1), Layer Network, Call Session Manager (CSM), Resource Manager (RM)

\section{CALL AND LAYER NETWORK}

The "call" is a unit of user's service request to service provider. The call in the B-ISDN environment has several requirements. Most of new services will be the multimedia multiparty services in future public networks, so the call should support these new services. To support multimedia services, it is necessary to define the basic components for each media that has its own property and they could be combined to cope with complex services. The multiparty 
services can not be viewed as just an extension of two-party services. The involvement of multiple parties in the same communication session may imply that the success of the establishment of a session is dependent on the existence of some specific party. Furthermore, each party can play different role. Therefore there should be capabilities to cope with these cases. There will be various quite different terminals in future networks. There will be telephones, cordless telephones, wireless telephones, workstations, personal computers, notebook computers, PDA (personal digital assistant)s, and so on. In this context, the problem of terminal and service capability will be significant and the call should support various terminals. There should be many resources to establish a call in B-ISDN environment, but there can be shortage of some resources, and it can cause another negotiation between user and service provider. So, it should be adapted to network circumstances and should be adapted for user's changing request during call setup phase.

Minzer introduced the "EXPANSE call model" that user can request multimedia multiparty services to network provider with extended transaction form. In the EXPANSE model, there are three kinds of elementary call objects. Those are local objects that are subject to control by a single user, confirmed objects that require concurrence with another user for construction and are subject to control by two users, and virtual objects that are created by network and may effect a user's behavior but are not directly subject to its control [1]. The EXPANSE call model is a basis of Bellcore's INA Communication Management Architecture [2]. There have been several researches of call model and resource model to provide multimedia multiparty communication [3][4][5].

The various functions comprising a telecommunications network can be divided into two broad classifications; delivery segment functions and service segment functions. Delivery segment functions are involved in the transfer of user's information. The delivery segment can be layered into a number of Layer Network (LN)s with a client-server relationship between adjacent layers. Each Layer Network represents the set of delivery segment that supports the transfer of a certain type of characteristic information. Examples of characteristic information include SONET/SDH VC-3 framed digital information, ATM cells, or Frame Relay blocks or fragments. Layering of the delivery segment offers several benefits to network providers in their tasks of operating and managing the network. A Layer Network can be partitioned according to several reasons; the network topology within a Layer Network, administrative boundaries between administrative authorities, domain boundaries, independent routing domain, etc. A Subnetwork can be recursively partitioned into a number of smaller Subnetworks interconnected by Links. There are many autonomous domains and the need for co-operation with other domains is increasing. The call that supports the layer networks should use resources of lower network efficiently and should hide the complexities of lower layer. [6]

TINA consortium is defining a software architecture for telecommunications systems. The mission of Connection Management functions in a TINA-C consistent network is to support telecommunication services in the need for connections. The TINA-C Connection Management Architecture (CMA) defines a target architecture for connection setup, control and release. The functionality of Connection Management is defined as a set of computational objects. The CSM (Communication Session Manager) provides services for the binding of computational stream interface. The clients of CSM will specify the references of stream 


\section{Layers Partitioning Within a Layer}

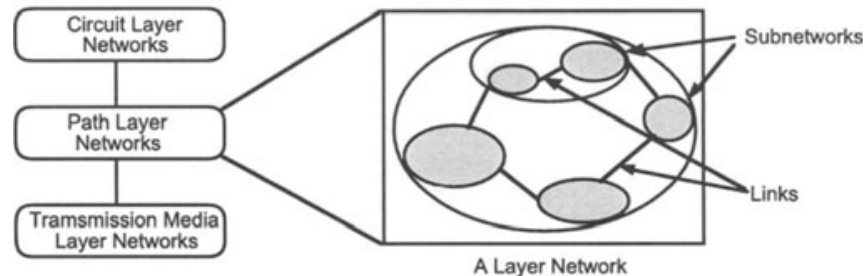

Figure 1 Layering and Partitioning [6].

interfaces they want to be interconnected, plus QoS parameters. The service of CC (Connection Coordinator) is the interconnection of physical nodes. The CC is responsible for creating connections between nodes. Connectivity through layer networks is provided by the collaboration of two types of computational objects: LNC (Layer Network Coordinator) and $\mathrm{CP}$ (Connection Performer). The LNC provides trails in a layer network. It also takes care of federation with other domains in a layer network. It requests the $\mathrm{CP}$ to set up the connection in its own domain. The CP provides connections in a single subnetwork. Since a subnetwork may be partitioned into smaller networks, each CP may request subordinate CPs to provide smaller subnetwork connections [7][8].

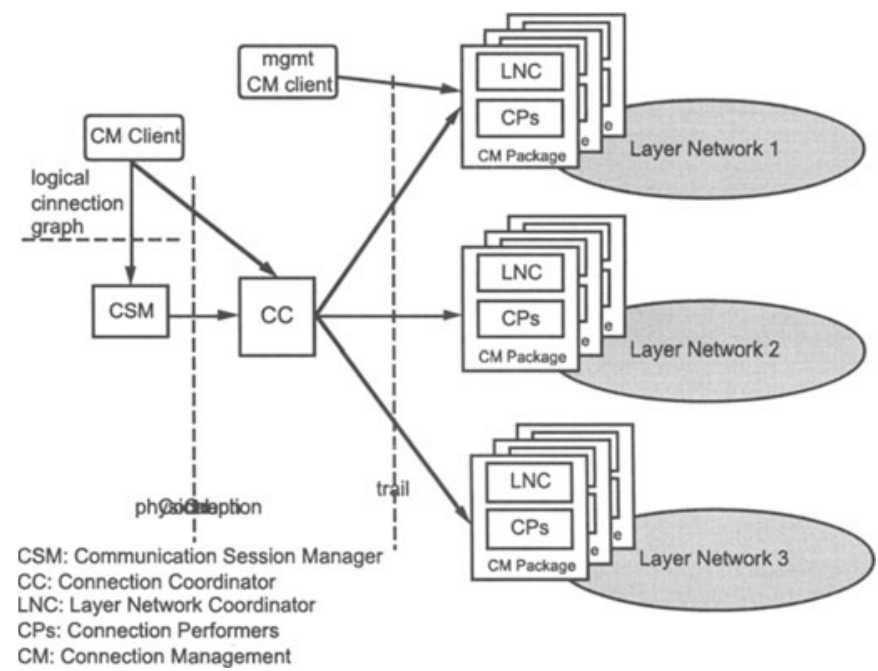

Figure 2 TINA-C's Connection Management Overview [8]. 
The TINA-C focuses on the communication management architecture and the communication management specification. However it does not consider the implementation of the CMA. In this paper, we propose a generic service call processing architecture: Service Call Processing Architecture 1 (SCPA1). The SCPA1 is well suitable for the Layer Network and it is a more practical architecture than TINA-C's CMA.

\section{SCPA1 (SERVICE CALL PROCESSING ARCHITECTURE 1)}

There are two functional elements to process a call in SCPA1. Those are elements to negotiate users' view and elements to manage resources of a lower layer. In the existing telephone service the user's requests are so simple that they can be mapped with the lower layer resources directly. When a user wants to use the telephone service he/she just picks up the phone and dials the destination telephone number. There is one connection per a call. However the user's requests in B-ISDN services will be very complex. He/she may want multimedia multiparty services. He/she can choose each medium's capabilities and each party's services.

There are the Call Session Manager (CSM) to receive and negotiate these user's complex requests and the Resource Manager (RM) manages lower layer resources. Figure 3 shows the SCPA1 that we propose.

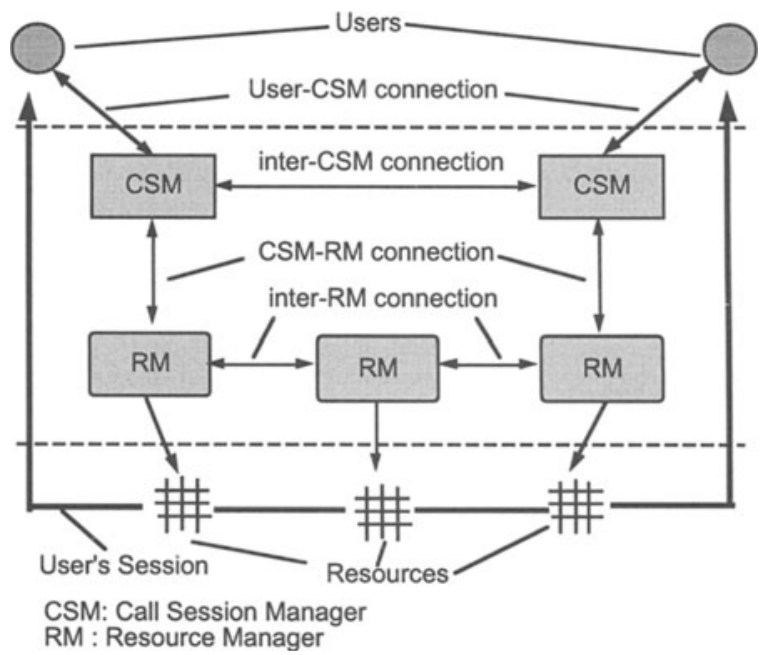

Figure 3 SCPA1: Service Call Processing Architecture 1 
There are 5 kinds of connections in SCPA1. Those are (1) the user-CSM connection, (2) the inter-CSM connection, (3) the CSM-RM connection, (4) the inter-RM connection, and (5) the user's communication session.

When a user wants to use the service, he/she should register at the CSM first. The CSM maintains the information of each subscriber (user's address, user's profile, address of the RM that provides connection to user, billing information, etc.) It may give him/her the information such as the current status of communication, the cost of current call, etc. The user-CSM connection is a control channel between user and service provider. When a user wants to use BISDN services, he/she may request them via the user-CSM connection. Though there is only a bearer service in the existing public network, there can be other services like data conversion, video compression, and so on in future network. So, the user should negotiate not only on the quality of services of the bearer service but also on the quality of service of these non-bearer services.

When the source CSM receives a user's request, it negotiates with the destination CSMs via the inter-CSM connection. There are two kinds of requests; one for adjusting both sides' semantics into the same and the other for using the lower layer's resources. The CSM should support negotiation of these two kinds of requests. The negotiation of a user's request can be performed as an atomic action or an interactive action. In the atomic action, a user sends the information of his/her communication to a destination and then the destination user determines the request and makes decision whether the call is accepted or not. However in the interactive action, the receiving user may also send the information of its communication environment and call is accepted after several information exchanges.

When the CSM receives the request from its user, it creates local objects and negotiation objects, and transfers the replication of the negotiation objects to the destination CSMs. The local object is the object that can be managed by one local CSM and the negotiation object is the object that should be managed by co-operation of CSMs. Each destination CSM creates the local objects, replicates negotiation objects, and notifies user of them. The receiving user can modify the request by modification of the negotiation objects. The CSM modifies its negotiation objects and passes this modification request to source CSM. Then the source CSM modifies its negotiation objects and notifies a user of them.

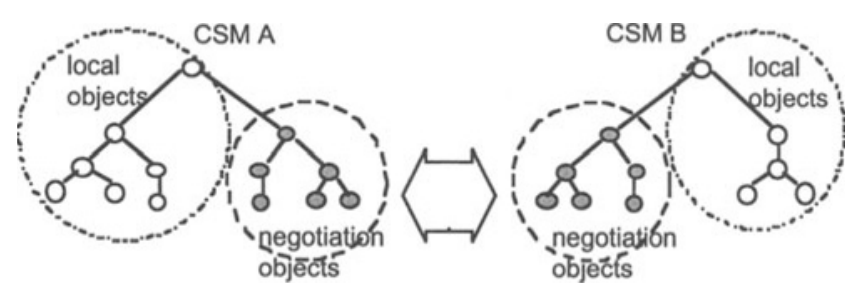

Figure 4 CSM objects 
After the negotiation, the CSM translates the user's requests to resources and requests the RM to perform the resource management functions via the CSM-RM connection. There are mediation objects to maintain the relationship between these CSM objects and the resources that are managed by the RM. There can be various relationships among these CSM objects and the RM objects. One CSM object can be related to several RM objects. Several CSM objects can also be related on one RM object. The mediation objects perform a mapping between CSM objects and RM objects.

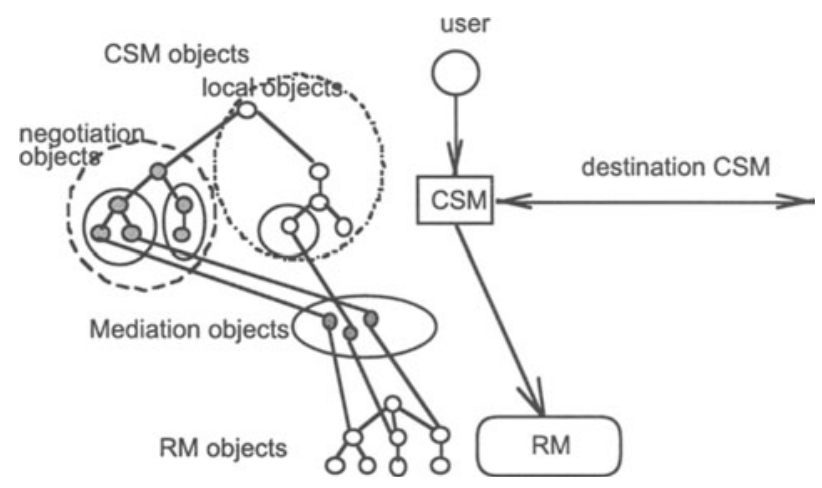

Figure 5 CSM objects, RM objects and Mediation objects

The RM can perform the resource management functions to its own resources. It can also request the tandem RM to perform the resource management functions. The RM is responsible for its domain. If a user wants to communicate with a user in an other domain, the RM should cooperate with other RMs. So, there can be one RM or several RMs in establishing one connection.

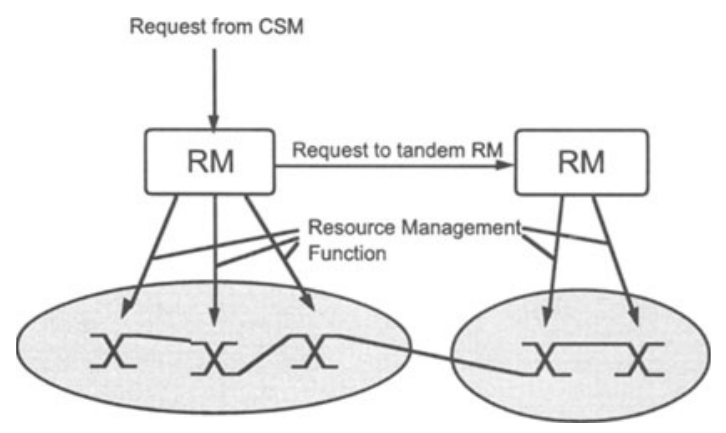

Figure 6 Role of RM 
Before the resource allocation, the RM reserves the resources first. Because the multimedia multiparty communication needs many resources, there can be a shortage of some resources. At that time, the RM notifies the CSM that the requested connection cannot be established, and the CSM does the negotiation again.

When all the resources are reserved, the RM notifies the CSM that the connection can be established. When all required connections can be established the CSM requests the RM to perform resource management functions for resource allocation. After all resources are allocated, the RM notifies the CSM that the connections are established. Then, the CSM announces to the user and the destination CSM that the session is ready.

\section{FUNCTIONAL MODELS OF THE CSM AND THE RM}

The functions of CSM can be decomposed into a number of fundamental functional groups, such as call session management functions (CSMFs) and system management functions (SMFs). There are 5 kinds of CSMF objects in CSM. Those are the access CSMF object, the object manager CSMF object, the signaling CSMF object, the routing CSMF object, and the mediation object manager CSMF object. Figure 7 shows the functional model of the CSM.

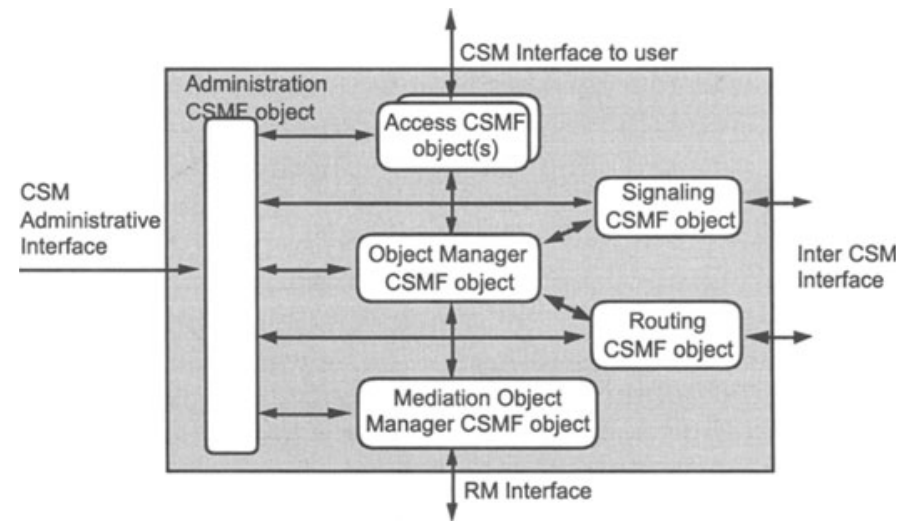

Figure 7 CSM Functional Model

The access CSMF object receives the user's requests and passes them to the object manager CSMF object. It also sends the notification from objects to upper users. There is one access CSMF object per one call. The object manager CSMF object processes the user's requests and it operates with the signaling CSMF object and the routing CSMF object to negotiate with other CSM's object manager CSMF objects. The signaling CSMF object terminates the net- 
work signaling between CSMs. The routing RMF object supports to create connections for signaling among CSMs. It determines a path between the source CSM and destination CSM. The mediation object manager CSMF object maps the CSM objects to RM objects.

The RM receives the request of the CSM and performs the lower layer's resource management function. One RM is responsible for its domain. Figure 8 shows the functional model of RM.

The access RMF object receives the CSM's requests and passes them to the object manager RMF object. It also sends the notification from resource object to the CSM. The object manager RMF object processes the CSM's request. It creates, deletes, modifies, and maintains logical resource objects. If the requested resources are in the RM's own domain, it performs lower layer resource management function via the lower layer resource RMF object. When the resource is not in its domain it co-operates with other $\mathrm{RM}$. When the resource is not in the layer, RM plays the user's role of the lower layer's CSM via the lower layer network RMF object.

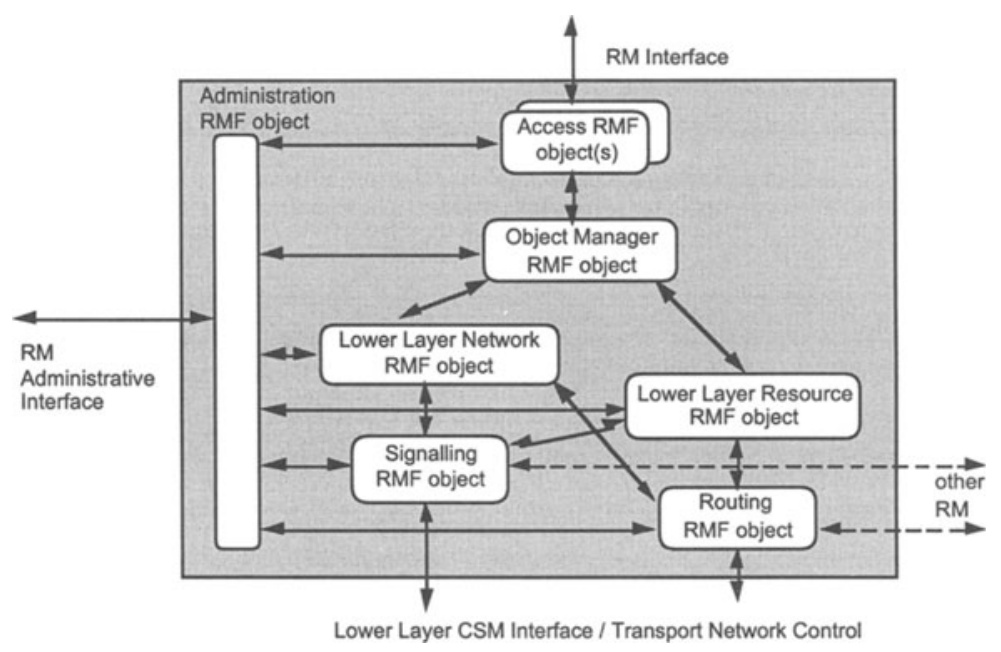

Figure 8 RM Functional Model

The signaling RMF object and the routing RMF object support the lower layer resource RMF object and the lower layer network RMF object. There are 3 kinds of signaling in the RM. One is for the lower layer resource management, another is for the cooperation between other RMs and the other is for the request to the lower layer network. The signaling RMF object terminates signaling between the RM and the CSM of the lower layer network and it also terminates signaling between the RM and the lower layer resources. Furthermore it terminates signaling between RMs. The routing RMF object determines 3 kinds of paths. It determines (1) paths between a source and destination address, (2) paths between RM and lower layer 
resources, and (3) paths between RMs. It also sends routing information to other RMs and receives it from others.

\section{CALL PROCESSING ALGORITHMS OF THE SCPA1}

We show the call processing procedure in this section. There are 4 phases in call processing in SCPA1. Those are the negotiation phase, the reservation phase, the allocation phase and the confirmation phase. The CSMs receive user's request and adjust both sides' views in the negotiation phase. After the negotiation the CSMs request the RMs to reserve resources. When all resources are reserved, the resource allocation phase is followed. Then the CSMs confirm to users that session is ready.

\subsection{Normal call processing}

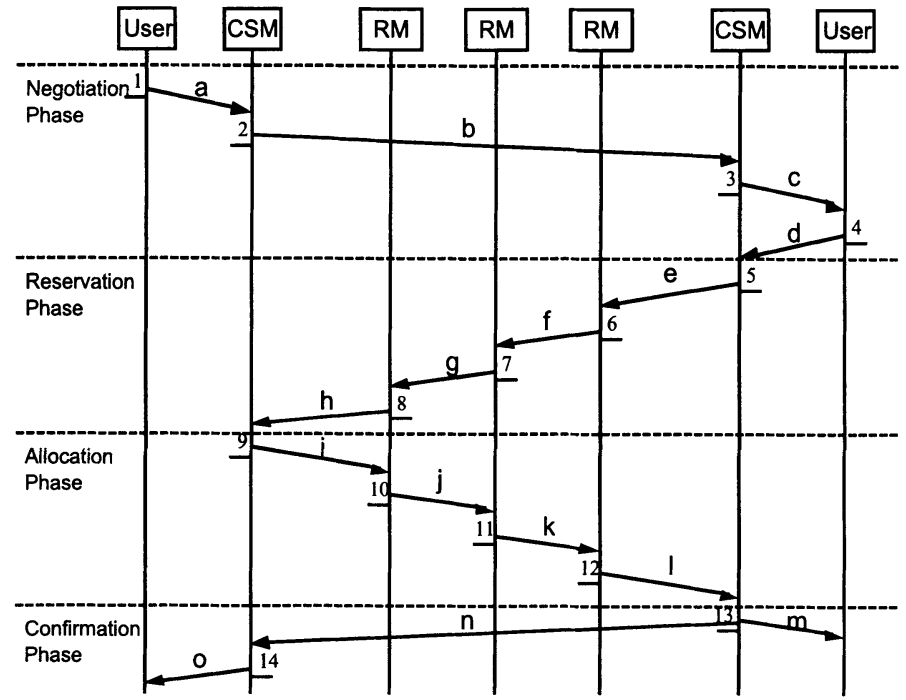

Figure 9 Normal Call Processing

The negotiation phase is from step 1 to step 4 .

Step 1: User A who wants to communicate with user B transfers the request to the CSM A. message a: $\{$ request to communicate with user $B$ \}

Step 2: The CSM A creates local objects and negotiation objects, and then transfers the request to the CSM B.

message $b:$ create negotiation objects \}

Step 3: The CSM B creates local objects and negotiation objects, and notifies its user of them. 
message c: \{ notify the creation of local objects and negotiation objects \}

Step 4: When user B wants to accept the call, he/she sends acceptance message to the CSM B. message d: $\{$ accept $\}$

The reservation phase is from step 5 to step 8 . In this phase, the CSM requests the RM to reserve the resources, and RMs do reservation.

Step 5: The CSM B requests the RM for resource reservation function. message $e:\{$ do resource reservation \}

Step 6,7: The RM reserves its own resources and requests the tandem RM to reserve the resources.

message $f, g:\{$ do resource reservation \}

Step 8: The RM reserves its own resources and notifies CSM A that all resources for the call are reserved.

message $h:\{$ all resources are reserved \}

The resource allocation phase is followed. In this phase, the RMs perform the resource management functions and request the tandem $\mathrm{RM}$ to perform it.

Step 9: The CSM A requests the RM to perform the resource allocation. message $i$ : $\{$ do resource allocation \}

Step 10,11: The RMs perform the resource allocation functions and request the tandem RM to perform the resource management functions.

message $j, k$ : $\{$ do resource allocation \}

Step 12: The RM B performs the resource management function and informs CSM B that it has performed the resource management functions successfully.

message $l:\{$ all resources are allocated \}

When all the resources are allocated, the CSMs notify users that the session is ready. We call this phase the confirmation phase.

Step 13: The CSM B notifies the CSM A and the user B that the session is ready. message $m, n:\{$ session is ready $\}$

Step 14: The CSM A notifies the user A that the session is ready. message $o:\{$ session is ready\}

\subsection{Refused call}

When a user requests unauthorized service to the CSM, the CSM can refuse the call. Because the destination CSM can do various functions such as authentication function, screen function, etc. it can also refuse the call. When the user B does not want to communicate with the user A, he/she can refuse it. Figure 10 shows the refused calls. 


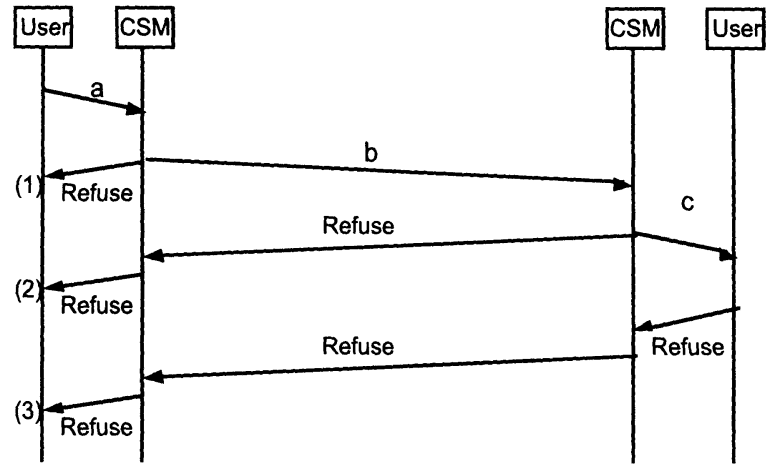

Figure 10 Refused Call

\subsection{Renegotiation of call}

If the user B wants to modify the attributes of negotiation objects, he/she requests the CSM to modify them. When the CSM receives this modification request, it modifies the negotiation objects and requests the peer CSM to modify them. After modification of its negotiation objects, the CSM notifies the user that the call is modified by the peer user. Figure 11 shows a 2way negotiation.

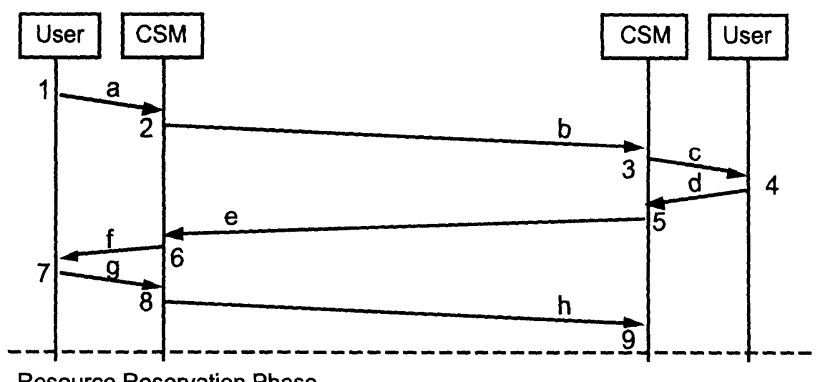

Figure 11 2-Way Negotiation

message d: \{ modify the negotiation objects \}

message e: \{modify the negotiation objects \}

message f: \{ notify the user of the modifications \}

message g: \{accept \}

message $h:\{$ accept $\}$ 
This negotiation can be repeated. Figure 12 shows a 3-way negotiation. If the user A does not agree the modification, he/she can also request to modify the negotiation objects, he/she requests the CSM to modify them. When the CSM receives this modification request, it modifies the negotiation objects and requests the peer CSM to modify them.

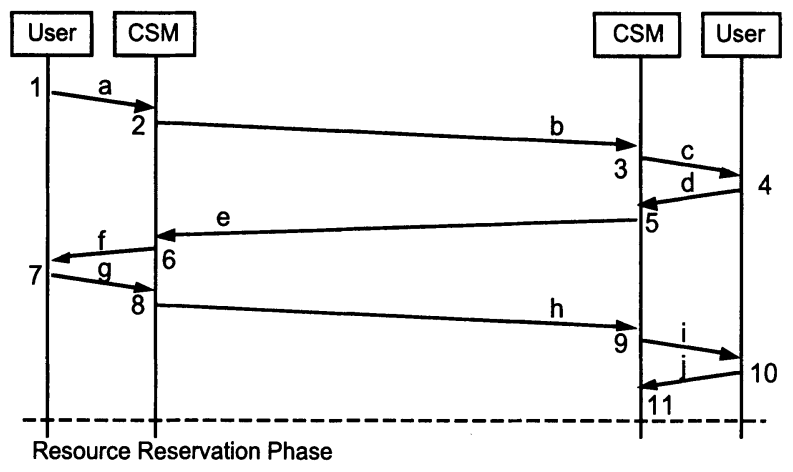

Figure 12 3-Way Negotiation
message d: \{ modify the negotiation objects \}
message $e:\{$ modify the negotiation objects \}
message f: \{ notify the user of the modifications \}
message $\mathrm{g}$ : \{ modify the negotiation objects \}
message $h:$ \{ modify the negotiation objects \}
message $i:\{$ notify the user of the modifications \}
message j: $\{$ accept $\}$

During the resource reservation phase, there can be a shortage of some resource. When it happens the RM notifies the requesting $R M$ of it, and the notified RM announces to the requesting RM. When the CSM receives this notification message, it should negotiate again.

\subsection{Call processing in a Layer Network}

Figure 13 shows the call processing in Layer Networks. You should notice that the negotiation phase of the lower Layer Network is in the resource reservation phase of the upper Layer Network. When the negotiation of the lower layer Network is completed, the upper layer's RMs continue the resource reservation. The resource allocation of the lower Layer Network is performed after the resource reservation phase of the upper Layer Network. 


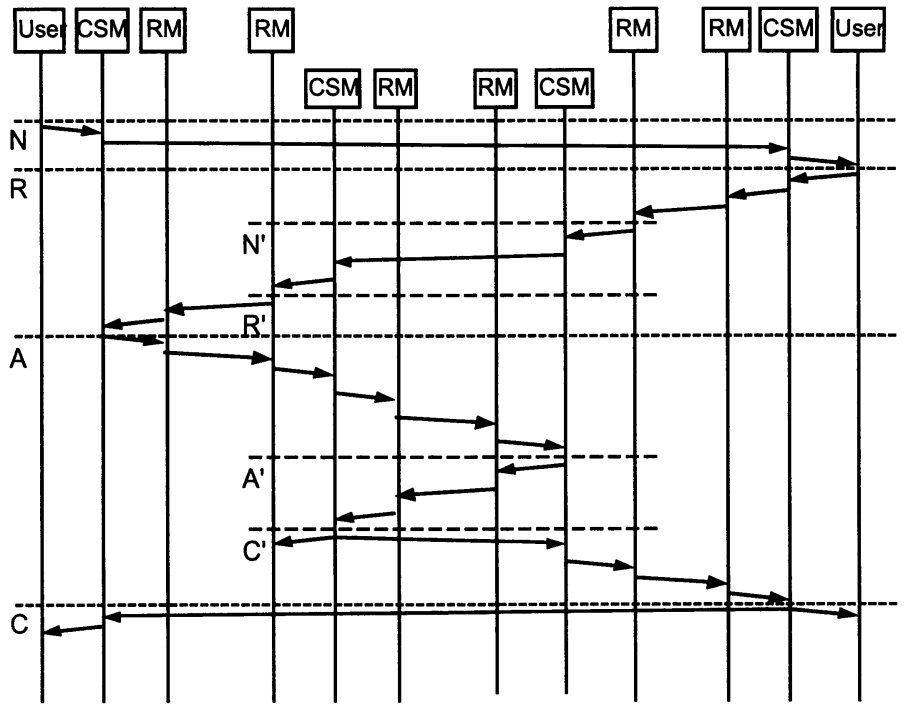

Figure 13 Call Processing in Layer Network

Figure 14 shows one example of the call processing. This shows the call processing in Layer Networks and the multiparty connections. There are 3 users and 3 CSMs in this figure, but one CSM is responsible for several users. There are 2 Layer Networks in this figure. You should notice that the negotiation process between the lower layer's CSMs. From the viewpoint of the upper layer's RM, the connection of the lower layer is a link. So, the RMs do not wait for the lower layer connection. 


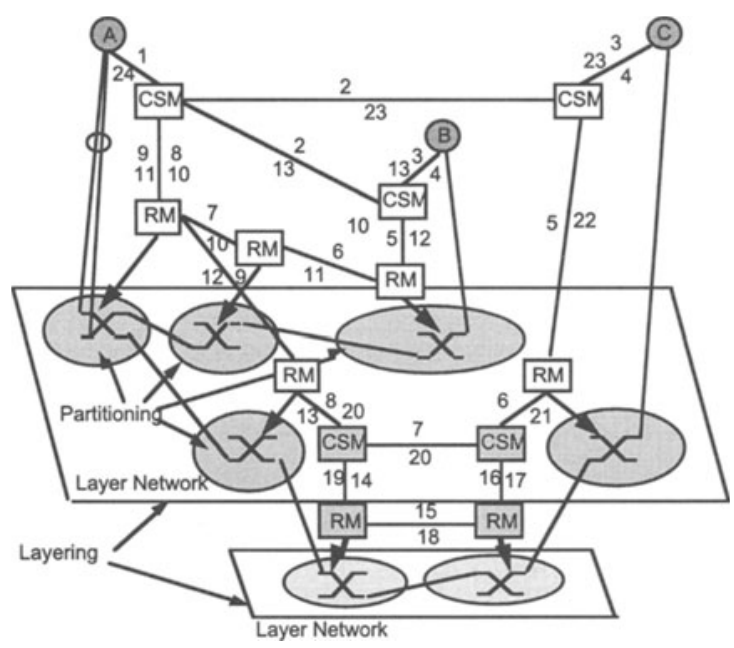

Figure 14 Example of Call Processing

\section{SUMMARY}

The Layer Network makes it possible to model the complex network composed of networks of several domains and several technologies. There will be new services in the future telecommunication network, and the most of them will be composed of existing services. The SCPA1 is a new call processing architecture for future networks, specially for the Layer Networks. We divide the call processing functions into two parts; one for negotiation of the upper layer's request and another for requesting the services of the lower layer. The CSM and the $\mathrm{RM}$ are responsible for performing those functions. We have presented the call processing algorithms and functional models of SCPA1. Now we have considered the generic resources of the service call and objects of each functional element.

\section{REFERENCES}

[1] S. Minzer, "A Signaling Protocol for Complex Multimedia Services," IEEE Journal on Selected Areas in Communications, Vol. 9, No. 9, Dec. 1991, pp. 1383-1394

[2] INA Document cycle 1 "Communication Management Architecture," Bellcore, 1992

[3] M. Jeffrey, A. Sarma, "A Signaling Architecture for Multimedia Services," Proc. of ICC'92 pp. 604-608

[4] M.E. Gaddis, R. Bubenik, J.D.Dehart, "A Call Model for Multipoint Communication in Switched Networks," Proc. of ICC'92, pp. 609-615 
[5] S.W.Tu, G.A.Brenner, "An Object-oriented Resource Model for Supporting Signaling and Control of Broadband Services," Proc. of ICC'92, pp. 616-621

[6] CCITT Study Group XVIII/7, Recommendation G.803, "Architecture of Transport Networks Based on the Synchronous Digital Hierarchy (SDH)"

[7] Martin Chapman, Stefano Montesi, "Overall Concepts and Principles of TINA," TB_MDC.018_1.0_94, 1995

[8] Motoharu Kawanishi, Hiroshi Oshigiri, Juan Pavon, Mike Schenk, "Connection Management Architecture", TB_JJB.005_1.5_94, 1995

\section{BIOGRAPHY}

Sunshin An was born in Seoul, Korea, in 1950. He received the B.Eng degree from the Seoul National University, Korea, in 1973, and the M.S degree in electrical engineering from the KAIST(Korea Advanced Institute of Science and Technology), Korea, in 1975, and the Doctor degree in electric and information from the ENSEEIHT, France, in 1979. He joined the faculty of Korea University in 1982, where he is currently Professor of Electronic Engineering. Prior to joining Korea University, he was Assistant Professor of Electronic Engineering in Aju University, Suwon, Korea. He was with NIST(National Institute of Standards and Technology) in U.S.A, as a Visiting Scientist, in 1991. His research interests include the distributed system, communication networks and protocols, information network, intelligent network and multimedia communication system. Dr. An is the Chair of OSIA(Open System Interconnection Association) in Korea, and the Secretary of the Korea Information Science Society. He is a member of the ACM and IEEE.

Geonung Kim was born in Korea in 1967. He received the B.Eng degree and the M.S degree in electronic engineering from the Korea University, Seoul, in 1990 and 1994, respectively. $\mathrm{He}$ is currently a graduate research assistant in Super Highway Information Network Laboratory at the Korea University, where he is pursuing his doctorate in electronic engineering. His interests include network management, TMN, intelligent network and information network. 